\title{
Un nouveau cas de choc anaphylactique induit par le rocuronium amélioré par le sugammadex
}

\section{A case of rocuronium-induced anaphylactic shock, improved by sugammadex}

\author{
Rachid Badaoui, MD • Ivan Popov, MD • \\ Hervé Dupont, MD
}

Received: 20 March 2012/Accepted: 17 May 2012/Published online: 1 June 2012

(C) Canadian Anesthesiologists' Society 2012

\section{Au rédacteur en chef,}

Depuis la mise en marché du sugammadex, plusieurs cas d'anaphylaxie au rocuronium ont été traités avec succès par le sugammadex..$^{1-3}$ Nous décrivons après consentement, le cas d'une patiente qui a subi une réaction anaphylactique grave au rocuronium à l'induction de l'anesthésie et qui a bénéficié d'une administration de sugammadex en plus du traitement traditionnel.

Une patiente de 52 ans $(77 \mathrm{~kg}$ pour $1,59 \mathrm{~m}$ ), était programmée pour le traitement chirurgical d'une tumeur rectale par laparoscopie. On notait dans ses antécédents un cancer du sein droit opéré, une hystérectomie totale et une césarienne. Il n'avait pas de notion d'allergie. Une antibioprophylaxie à la céfazoline $2 \mathrm{~g}$ est administrée 30 min avant le début de l'anesthésie. L'induction de l'anesthésie se réalise par l'injection de propofol $200 \mathrm{mg}$ et de sufentanil $20 \mu \mathrm{g}$. Après vérification d'une ventilation manuelle sans difficulté, une injection de rocuronium $50 \mathrm{mg}$ est administrée. Deux minutes plus tard, on constate une tension artérielle systolique à $50 \mathrm{mmHg}$ avec tachycardie à 150 battements $\min ^{-1}$, puis rapidement une tension artérielle imprenable au brassard avec un érythème généralisé, une désaturation, une baisse de la concentration télé-expiratoire de $\mathrm{CO}_{2}$ à $15 \mathrm{mmHg}$. Après intubation de la trachée avec ventilation manuelle en oxygène pur et un remplissage vasculaire $(500 \mathrm{~mL}$ d'hydroxyéthylamidon et $500 \mathrm{~mL}$ Ringer lactate), une administration intraveineuse d'adrénaline en doses successives de $0,1 \mathrm{mg}$ est débutée avec un relais à $0,5 \mathrm{mg} / \mathrm{h}$ en continu. Un bilan sanguin est prélevé en même temps. Vingt minutes après le début de la réanimation et malgré des doses supplémentaires

R. Badaoui, MD $(\varangle) \cdot$ I. Popov, MD · H. Dupont, MD

CHU Nord, Amiens, France

e-mail: badaoui.rachid@chu-amiens.fr d'adrénaline (6 $\mathrm{mg}$ au total) et un remplissage $\left(30 \mathrm{~mL} \cdot \mathrm{kg}^{-1}\right)$, la tension artérielle reste à $70 \mathrm{mmHg}$ avec apparition d'une bradycardie à 35 battements. $\min ^{-1}$ et de quelques troubles de rythme. On décide alors d'injecter du sugammadex $1000 \mathrm{mg}$ dans l'hypothèse d'un choc anaphylactique dû au rocuronium, en espérant un bénéfice dans cette situation menaçant le pronostic vital. Cinq minutes plus tard, on note un pouls carotidien et radial, une restauration hémodynamique, ainsi qu'une recoloration cutanée. Sous adrénaline $0.7 \mathrm{mg} \cdot \mathrm{h}^{-1}$, la tension artérielle est alors à $85 / 46 \mathrm{mmHg}$ et la fréquence cardiaque à 97 battements. $\mathrm{min}^{-1}$. La patiente reçoit du midazolam comme sédation pour la mise en place d'une canule dans l'artère radiale gauche et d'un cathéter veineux central dans la jugulaire interne droite. La patiente est sevrée en catécholamines une heure après son admission en unité de surveillance continue. La trachée est extubée après arrêt de la sédation. La tension artérielle est alors à 103/47 mmHg et le pouls à 103 battements. $\mathrm{min}^{-1}$. La patiente quitte l'hôpital après $48 \mathrm{~h}$. Le bilan biologique donne les résultats suivants: histamine $>100 \mathrm{nmol} \cdot \mathrm{L}^{-1}\left(\mathrm{~N}<10 \mathrm{nmol} \cdot \mathrm{L}^{-1}\right)$; tryptase: $102 \mu \mathrm{g} \cdot \mathrm{L}^{-1}\left(\mathrm{~N}<11,4 \mu \mathrm{g} \cdot \mathrm{L}^{-1}\right)$; IgE spécifique au rocuronium: 46,33 \% d'inhibition (positif). Les tests cutanés (pricks tests) sont positifs pour le rocuronium avec une papule de $8 \mathrm{~mm}$ de diamètre associée à un prurit.

Depuis la publication du cas clinique rapporté par McDonnell et coll., ${ }^{1}$ qui représente la première illustration de l'intérêt potentiel d'une administration de sugammadex en cas de réaction anaphylactique réfractaire aux catécholamines induit par le rocuronium, plusieurs publications ont fait état d'observations semblables. ${ }^{2,3}$ Puisque des études randomisées sont impossibles, l'évaluation de l'intérêt potentiel de telles approches thérapeutiques ne pourra se faire qu'à travers la publication de cas cliniques, qu'ils soient positifs ou négatifs. Dans 
notre cas, la patiente a développé un choc anaphylactique de grade III après l'administration du rocuronium et les symptômes ont été complètement résolus après l'administration du sugammadex en plus de la réanimation traditionnelle. Les raisons de cette amélioration sont peu claires. $^{4,5}$

\section{Conflit d'intérêt Aucun.}

\section{Références}

1. McDonnell NJ, Pavy TJ, Green LK, Platt PR. Sugammadex in the management of rocuronium-induced anaphylaxis. $\mathrm{Br} \mathrm{J}$ Anaesth 2011; 106: 199-201.
2. Raft J, Leclercq M, Longrois D, Meistelman C. Récupération hémodynamique et ventilatoire rapide après injection de sugammadex lors d'un choc anaphylactique au rocuronium, réfractaire au traitement conventionnel. Ann Fr Anesth Reanim 2012; 31: 158-61.

3. Motamed C, Baguenard P, Bourgain JL. Possible mitigation of rocuronium-induced anaphylaxis after administration of sugammadex. J Anaesthesiol Clin Pharmacol 2012; 28: 127-8.

4. Leysen J, Bridts CH, De Clerck LS, Ebo DG. Rocuronium-induced anaphylaxis is probably not mitigated by sugammadex: evidence from an in vitro experiment. Anaesthesia 2011; 66: 526-7.

5. Jones PM, Turkstra TP. Mitigation of rocuronium-induced anaphylaxis by sugammadex: the great unknown. Anaesthesia 2010; 65: 89-90. 